\title{
REFLEXIONES AL FINAL DE UN INICIO: ESTUDIO DE ASOCIACIÓN ENTRE VARIABLES SOCIODEMOGRÁFICAS Y TIPO DE PARTO EN LA PREVENCIÓN DE LA VIOLENCIA OBSTÉTRICA EN ESPAÑA'
}

\section{Cristina Benlloch-Doménech ${ }^{1}$, María José Barbé-Villarubia² ${ }^{2}$ Jorge Cascales Ribera $^{3}$}

Autora correspondencia: Cristina Benlloch-Doménech

Correo electrónico: cristinabenlloch@gmail.com

1. Doctora en Ciencias Sociales por la Universidad de Valencia (UVEG). Ayudante doctora. Departamento de Sociología y Trabajo Social de la Facultad de Ciencias Sociales de la Universidad de Valladolid (UVa), Segovia.

2. Trabajadora Social. Personal Investigador en Formación del programa «Atracción de Talento» (ref: INV17-01-13-18) de la Universidad de València vinculada al programa de doctorado en Ciencias Sociales de la Facultad de Ciencias Sociales de la Universidad de Valencia (UVEG), Valencia.

3. Educador Social. Profesor Asociado. Departamento de Métodos de Investigación y Diagnóstico en Educación de la Facultad de Filosofía y Ciencias de la Educación de la Universidad de Valencia (UVEG), Valencia.

Recibido: 30/11/2018 Aceptado: 18/01/2019

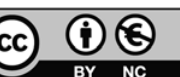

${ }^{1}$ Este trabajo fue presentado previamente como comunicación en el III Congreso Internacional Género, Ética y Cuidado «Visibilizando la violencia hacia las mujeres en el embarazo», celebrado en Barcelona en mayo de 2018. 


\section{RESUMEN}

\section{Objetivo}

El objetivo de nuestro trabajo es realizar una aproximación desde el punto de vista sociodemográfico a la forma y técnica de finalización del embarazo en el momento del parto, especialmente en cuanto al porcentaje de cesáreas.

\section{Material y métodos}

Se han analizado los datos de nacimientos en España recogidos por el Instituto Nacional de Estadística.

\section{Resultados}

Los resultados obtenidos en el cruce de variables muestran que existen determinantes sociales que harían comprender mejor los procesos de parto.

\section{Discusión}

La perspectiva aportada va más allá de la descripción y pretende mostrar una causalidad a ser tenida en cuenta en los protocolos obstétricos.

\section{Conclusiones}

El personal sanitario debe entender las circunstancias personales de las pacientes para que el parto se desarrolle con la mayor facilidad posible, atendiendo a las características de la mujer y no tanto a la preocupación por el porcentaje de cesáreas resultantes.

Palabras Clave: cesáreas; factores sociales; análisis; violencia obstétrica; nacimiento. 


\section{ABSTRACT}

\section{Objectives}

The aim of our work is to approach types of childbirth at the end of pregnancy from a sociodemographic point of view, with special emphasis in the number of $\mathrm{C}$ sections.

\section{Material and Method}

The birth data in Spain provided by the National Institute of Statistics has been analyzed.

\section{Results}

The results obtained from crossing the variables show that there are social determinants that should be considered in order to better understand the process of childbirth.

\section{Discussion}

Our approach goes beyond the mere description and aims to show a causality that should be included in obstetric protocols.

\section{Conclusion}

Health workers must be aware of the personal circumstances of the patients so that the delivery develops as easily as possible, taking into account the characteristics of the woman, and being not so much concerned with the number of cesarean sections they have to perform.

Keywords: cesarean; social factors; analysis; obstetric violence; birth. 


\section{INTRODUCCIÓN}

Del total de partos ocurridos en España en el año 2015, más del $26 \%$ terminaron en cesárea (la cifra más elevada se encuentra en Chipre con el 51\%) (Hadjigeorgiou et al, 2018). Ya en el año 1985, la OMS (Organización Mundial de la Salud) advertía que el índice de cesáreas debería estar en torno al $10 \%$ o el $15 \%$ del total de partos, aunque en su discurso más reciente no se fija un porcentaje y afirma que debe reducirse al menor número posible, sin determinar una cifra concreta. En este sentido, en los últimos años, esta tasa de cesáreas se ha incrementado, a pesar de las indicaciones que el organismo internacional viene dando desde hace décadas (ver Gráfico $n^{\circ} 1$ ). En lo que respecta a los centros donde se producen un mayor número de cesáreas, cabe destacar que, en los hospitales privados, el porcentaje es mayor.

Este incremento de las cesáreas no es un caso aislado en España, ni mucho menos reciente. En la década de los ochenta, ya se encuentran estudios que plantean cuestiones acerca del incremento de este desenlace del embarazo (Bottoms, Rosen y Sokil, 1980; Márquez-Calderón et al., 2011). En relación a las estadísticas de partos por cesárea de los últimos años, en España, en el primer y en el segundo año de la serie, el porcentaje del total de cesáreas se encontraba por encima de la recomendación de la OMS (entre el 10 $\%$ y el $15 \%$ ). Sin embargo, en el año siguiente y durante el resto de la serie, el porcentaje de intervenciones quirúrgicas era ya de uno de cada cuatro partos.

No obstante, encontrar una justificación no es tan simple, y es por ello por lo que dedicamos el presente estudio a comprender si existen factores de corte sociodemográfico, como la provincia de residencia, el nivel de estudios o el tipo de trabajo, que pueden llevar aparejada una mayor probabilidad de tener un parto que finalice con cesárea.

Este es un momento en el que el retraso de la maternidad hace que las mujeres sean cada vez mayores a la hora de dar a luz, y que esto suponga un incremento de las cesáreas, puesto que «parece» probado que, a mayor edad, más probabilidad de intervención quirúrgica. Así lo establecen autores como Blanca Heras et al. (2011), Oswaldo TapianiRodríguez (2005), o Anna Kristell Nolasco-Blé (Nolasco-Ble, Hernández-Herrera y Ramos-González, 2012).

Nuestro país es uno de los lugares donde la edad de las madres al tener el primer hijo es más elevada, muy próxima a los 32 años, y la tasa de cesáreas se incrementa con la 
edad. Es por ello, quizás, por lo que la OMS no habla de una cifra cerrada de cesáreas, sino que se limita a decir en el menor número posible, por la entrada en el escenario de nuevos factores asociados a los partos por cesárea que antes no estaban tan presentes.

Gráfico 1. Evolución del porcentaje de cesáreas por año

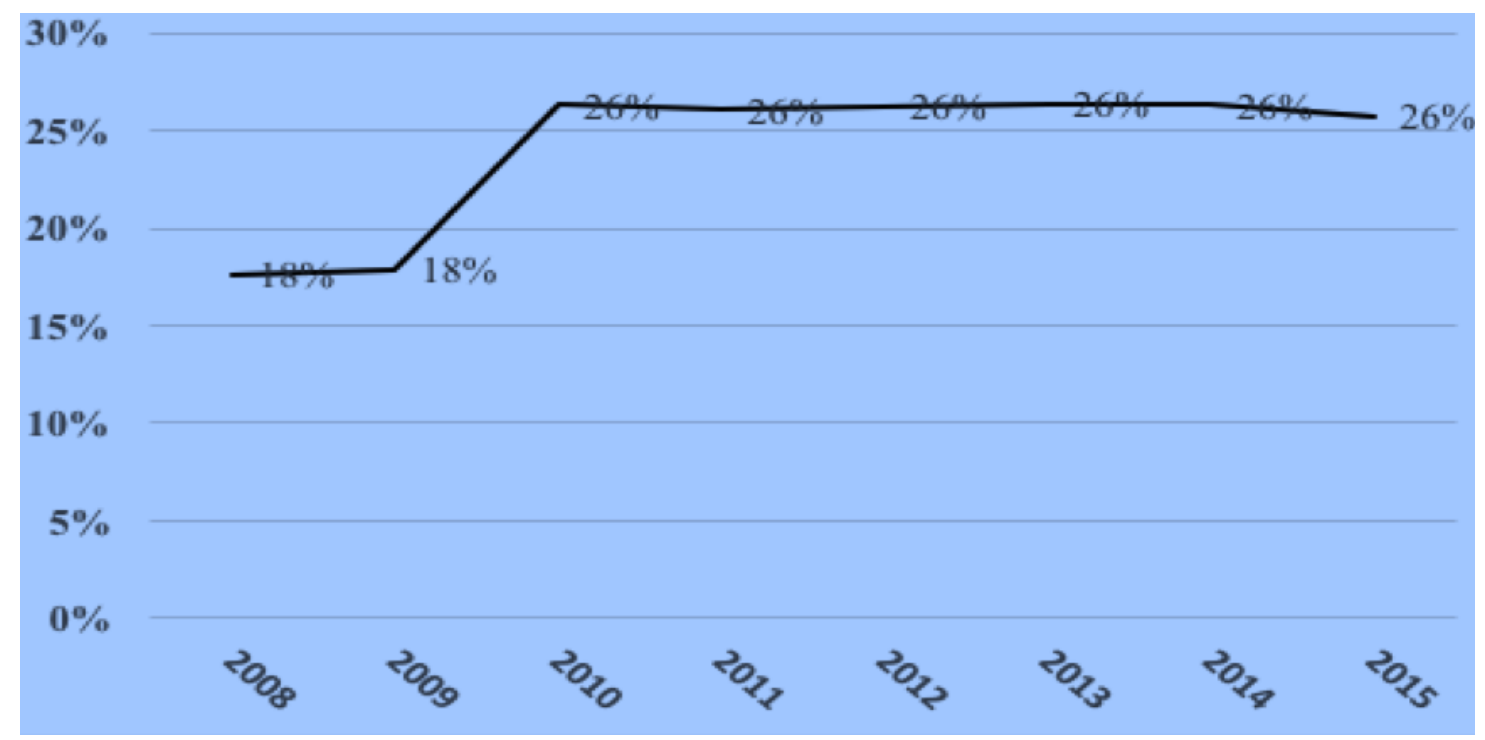

Fuente: Elaboración propia a partir de datos del INE

Gracias a la modernización de la medicina y los protocolos hospitalarios, sobre todo en los países occidentales, se ha reducido la mortandad en los partos, tanto de las madres como de los y las niños/as. Sin embargo, las mujeres, en este proceso, han ido perdiendo poder sobre sus propios cuerpos (Al Adib Mendiri et al., 2017; García, 2017). Este proceso se ha ido dando paulatinamente, centrándose en un criterio científico-médico (Al Adib Mendiri et al., 2017) y generando un cambio en el proceso de maternidad a lo largo de los últimos tiempos.

En la antigüedad era un proceso privado, puesto que se daba a luz en el seno de los hogares, siempre acompañadas de otras mujeres (como las matronas), mientras que, en la actualidad, se trata como una patología en los centros sanitarios, donde las mujeres son asistidas por profesionales (Al Adib Mendiri et al., 2017). De este modo, los asuntos relacionados con todo el proceso de embarazo y luego el parto son considerados asuntos «intra-expertos» (Villarmea, Olza y Recio, 2015). Será en el siglo XX cuando este proceso se institucionaliza y se empieza a considerar el embarazo y el parto como experiencias vitales a tener en cuenta desde el ámbito sanitario especializado, sobre todo este último entendido como un proceso médico (Gijón, 2016). 
En este proceso, desde los movimientos de mujeres y movimientos de reivindicación de los derechos de las mujeres, se ha acuñado el término «violencia obstétrica» como forma de visibilización de una violencia que sufren las mujeres en los procesos de embarazo y parto. En el debate, existe una diversidad de prácticas que son rechazadas y otras reclamadas por las mujeres, previamente y durante el parto. Una figura que está surgiendo en este debate sobre la forma en la que se atiende el parto es la doula: Se trata de una figura de mujer acompañante que está siendo cuestionada por representantes de la comunidad médica, pero que llena el vacío que muchas mujeres sienten en el proceso medicalizado de sus partos. ${ }^{2}$

La violencia obstétrica es un tipo de violencia basada en el género que implica la pérdida de poder sobre el propio cuerpo de las mujeres en los procesos de embarazo y parto y que, además, implica un abuso injustificado de la medicalización. Es un trato degradante y deshumanizador que, como consecuencia, deriva en la pérdida de autonomía de las mujeres y de su poder de decisión durante todo el proceso (Bellón, 2015).

Por otra parte, y como señalan otras autoras, limitar este concepto a la violencia que se ejerce sobre el cuerpo de las mujeres en el proceso de embarazo y parto es reduccionista. De este modo, se puede ampliar este tipo de violencia a la violencia que se ejerce sobre los cuerpos de las mujeres y su salud reproductiva a lo largo de toda su vida, estén embarazadas o no (Al Adib Mendiri et al., 2017).

Entre otras, cabe destacar las siguientes actuaciones como actos de violencia obstétrica ejercidos sobre las mujeres por parte de los especialistas en el momento previo y durante el parto (Fernández Guillen, 2015):

- Impedir pensar en el parto.

- No ofrecer alternativas para poder escoger.

- Separación de los hijos e hijas al nacer.

- Privar el apoyo de la persona que se elija como acompañante en el proceso de parto.

\footnotetext{
${ }^{2}$ Las doulas son personas, mayoritariamente mujeres, que acompañan durante el parto a la persona gestante antes y en el momento de dar a luz. Realizan la función de parteras acompañantes, las cuales han sido muy criticadas porque no suelen tener formación reglada o especializada para la realización de dicho acompañamiento. Esta práctica es típica de países de centroamérica y sudamérica, aunque actualmente, ante las carencias de atención individualizada en los hospitales, se está desarrollando también en España. Ver: https://www.eldiario.es/sociedad/parteras-aprovechan-lagunas-sistema-sanitario_0_357314943.html.
} 
- Inducir partos por conveniencia.

- Falta de intimidad en el momento del parto.

- Práctica de la episiotomía.

Como ponen de relieve muchas autoras, este tipo de violencia es una vulneración de los derechos humanos, así como de los derechos reproductivos.

Gracias a este concepto y a la lucha de grupos de mujeres por la eliminación de este tipo de violencia, se han denunciado algunos protocolos que no respetan las recomendaciones de los organismos internacionales. La propia OMS recomienda los partos naturales sin inducciones y sin cesáreas si no son necesarias, y sin infringir la intimidad de la propia mujer, teniendo en cuenta su derecho a decidir, así como la elección de la postura y la no restricción de agua (Bellón, 2015; Da Silva Carvalho y Santana, 2017).

Según la OMS: «Esta medicalización creciente de los procesos de parto tiende a debilitar la capacidad de la mujer de dar a luz y afecta de manera negativa su experiencia de parto. Además, el mayor uso de intervenciones en el trabajo de parto sin indicaciones claras continúa ampliando la brecha sanitaria en cuanto a la equidad entre entornos con muchos recursos y aquellos con pocos recursos» (2018).

Por otra parte, hay otros organismos que reconocen el derecho de las mujeres a una mejor salud sexual y reproductiva y, aunque la mayoría de veces interpretemos esto aplicándolo a los países empobrecidos y al gran problema de mortandad infantil que en ellos existe, también podemos aplicarlo ahora a los países en los que se está patologizando $\mathrm{y}$, por tanto, medicalizando en exceso el proceso de embarazo y parto.

Como bien es conocido, en septiembre del año 2000, se celebró en Nueva York la cumbre del milenio, en la que se aprobó la declaración del milenio. En esta declaración, los países de la Naciones Unidas, a través del Programa de Naciones Unidas para el Desarrollo (PNUD), se comprometían a implementar una serie medidas con el fin de mejorar las vidas de las personas para el año 2015, mediante los Objetivos de Desarrollo del Milenio (ODM). Entre los ocho objetivos que se establecieron, encontramos uno que nos interesa especialmente para este trabajo, el de «mejorar la salud materna». También cabe destacar que se establecen la igualdad de género y el empoderamiento de las mujeres como ejes transversales para poder alcanzar dichos objetivos. 
En septiembre de 2015 se celebró de nuevo otra cumbre, esta vez para establecer los objetivos después de 2015, y es aquí donde se elaboró la «Agenda 2030 para el Desarrollo Sostenible». Desde la perspectiva de género, esta nueva agenda supone un avance en materia de igualdad para las mujeres, ya que se recoge específicamente como objetivo «lograr igualdad de género y empoderamiento para todas las mujeres y niñas». Esto significa que dicho objetivo no solo va a tener una implementación formal, sino que se va a dotar de recursos para su consecución. Además, en este mismo objetivo, se desarrollan una serie de metas, entre las que encontramos la siguiente: «Garantizar el acceso universal a la salud sexual y reproductiva y los derechos reproductivos, de conformidad con el Programa de Acción de la Conferencia Internacional sobre la Población y el Desarrollo, la Plataforma de Acción de Beijing y los documentos finales de sus conferencias de examen» (PNUD, 2015).

Cuando la violencia obstétrica queda patente, se trata de una auténtica violación de los derechos humanos, tanto desde el enfoque de la violencia contra las mujeres, como desde la protección de la salud como derecho humano (Al Adib Mendiri et al., 2017).

A nivel nacional, en el estado español, no hay una tipificación en el código penal, pero este tipo de violencia viola varios derechos fundamentales recogidos en la constitución española, como el recogido en el artículo 15, «derecho a la integridad física y moral», en el artículo 18, «derecho a la intimidad», y en el artículo 17, «libertad personal» (Al Adib Mendiri et al., 2017).

En los últimos años, se ha dado un paternalismo médico entre profesional-mujer, en el que en la mayoría de los casos se realizan prácticas sin tener en cuenta la opinión de la mujer, lo que conlleva a una pérdida de su poder de decisión respecto a su propia salud sexual y reproductiva (Al Adib Mendiri, 2017).

Por ello, en los años 80, aparece por primera vez en los EEUU el llamado «plan de parto» como una herramienta que pueda dar solución a este paternalismo para facilitar la comunicación entre las mujeres y el personal que las atiende en estos procesos, aunque en España este documento no llega hasta una década más tarde. Este plan de parto es un documento elaborado por la propia mujer en el que puede expresar sus preferencias sobre el proceso de parto y nacimiento (Gijón, 2016).

Como bien sabemos, no tener en cuenta la voz o la opinión de las pacientes puede ser producto de acciones y decisiones que puedan generar en una amplia diversidad de 
malestares, tanto de forma implícita como explícita, tanto de forma consciente como inconsciente, causando violencia obstétrica en la relación médico-mujer. Este tipo de violencia es una forma de control social. Dado que la mayoría de los médicos especialistas son hombres, se sigue el patrón de control social hombre-mujeres, además de, paralelamente, el patrón de control-subordinación médico-paciente (García, 2017).

De esta forma, ya centrándonos en el momento de dar a luz, el paritorio es el escenario ideal para recrear esas relaciones de poder-subordinación, dada la circunstancia emocional de la preocupación porque todo salga bien, ya que está en juego la vida de la madre y la del propio recién nacido/a (Fernández Guillén, 2015).

A pesar de todo esto, cabe destacar que algunos países de Latinoamérica han sido pioneros en legislar en esta materia, y de introducir en sus leyes el término «violencia obstétrica», como por ejemplo Venezuela, el primer país del mundo en legislar en este sentido en el año 2007.

Después fue Argentina, en el año 2009, y progresivamente se ha ido incorporando en algunos estados mexicanos hasta el año 2012, cuando el senado nacional de México lo incluyó en varias leyes sobre violencia contra las mujeres (Bellón, 2015; Al Adib Mendiri, 2017; García, 2017).

Para finalizar, como anotan Cerdán-Jiménez et al. (2017), es importante trabajar con las mujeres un verdadero plan de parto e intentar que, en la medida de lo posible, se dé una humanización del proceso de parto de modo que la asistencia se centre en el bienestar de la mujer. Esto no es simplemente sinónimo de parto natural, puesto que, obviamente, un parto puede precisar de intervención médica pero no por ello el personal médico puede dejar de velar porque la gestante y su familia se sientan respetados e informados en todo momento.

Por todo ello, tal como hemos manifestado previamente, nuestro trabajo tiene como objetivo ahondar en las causas sociales del incremento de cesáreas, para analizar si los protocolos que se desarrollan para evitar la cesárea terminan por causar un «mayor daño en las mujeres» del que se pretendía evitar. Asimismo, queremos poner en cuestión si se da o no la decisión de las mujeres sobre el tipo de parto que desean. Nuestra hipótesis de trabajo pretende dar a entender que cada mujer es diferente, y que los protocolos están bien, pero se trabaja con personas en un momento crucial donde participan un gran 
número de emociones, así como factores derivados de la socialización diferencial, que pueden desembocar en violencia obstétrica.

\section{MATERIAL Y MÉTODO}

Este artículo es una aproximación desde el punto de vista estadístico a la base de datos de nacimientos recogida por el INE (Instituto Nacional de Estadística de España), elaborada a partir de las fichas que se rellenan en los hospitales con todas madres que han dado a luz en España. Esta estadística posee la ventaja de actuar como un censo de los nacimientos porque se trata una herramienta fundamental para el análisis estadístico y con poco margen de error.

Tras la revisión bibliográfica realizada, el análisis que se plantea es la existencia de factores de corte sociodemográfico en los partos con cesárea. Para esto, se ha realizado un análisis estadístico bivariante en el que se cruza el tipo de parto con las demás variables (nivel de estudios, profesión de la mujer, localización provincial del parto con cesárea), un primer paso que permitirá posteriormente una aproximación más compleja. En concreto, se ha realizado el Test Chi Cuadrado de Pearson para medir si existen diferencias o no entre las variables descritas y el tipo de parto. El cruce de variables se realizó con el programa estadístico SPSS.

Se optó por el nivel de estudios y el trabajo de la mujer porque son dos variables determinantes para la reproducción. Sabemos que, con la incorporación de la mujer al mercado laboral, se redujeron las tasas de maternidad en España desde la segunda mitad del siglo XX. En la actualidad, el incremento del nivel de estudios de las mujeres muestra un retraso en la fertilidad. Además, trabajo y formación condicionan la independencia de las mujeres y es una de las bases para el empoderamiento femenino.

El índice de las cesáreas no es importante en sí, sino por el hecho de que enmascara situaciones de violencia médica, a pesar del cambio institucional, social y científico que se ha producido en los centros médicos. Del mismo modo, es importante porque obvia los condicionantes sociales que afectan a la salud, tratando a todas las mujeres «por igual» a pesar de sus diferencias. 


\section{RESULTADOS}

En lo que respecta a las variables, el nivel de estudios resultó estadísticamente significativa. Las mujeres que presentan el porcentaje menor de cesáreas es el de las mujeres sin estudios, entre las cuales las cesáreas son el $22 \%$ de los partos (Joseph et al., 2003); mientras que entre las que tienen la licenciatura, el resultado es del $28 \%$, por lo que existe más de 6 puntos porcentuales de diferencia. En lo que respecta a la significatividad estadística, al realizar el coeficiente de contingencia, este muestra que existe una relación significativa entre las dos variables.

Para analizar todas las relaciones entre las variables, se realizó tanto el chi cuadrado como el coeficiente de contingencia. En el caso de la relación entre la variable nivel de estudios más tipo de parto, se muestra que las variables tipo de parto (cesárea o parto natural) es dependiente del nivel de estudios de la mujer.

En consecuencia, podemos decir que el nivel de estudios de las mujeres que vayan a proceder al parto debería ser una característica a tener en cuenta para configurar un mejor perfil de las mujeres con la finalidad de prevenir o preparar con antelación a la gestante ante una situación de parto con cesárea, en tanto que la formación en estudios superiores puede llegar a ser un factor por el cual se retrase el momento de decidir tener hijos/as, pudiendo ser otra marca que correlacione directamente con la edad de la gestante y las cargas que aparecen con el acceso al mercado de laboral.

Tabla 1 Test de Chi Cuadrado de Pearson para la relación entre las variables nivel de estudios y cesárea

Pruebas de chi-cuadrado

\begin{tabular}{|c|c|c|c|}
\hline & Valor & Gl & Sig. asintótica (2 caras) \\
\hline Chi-cuadrado de Pearson & $467,623^{\mathrm{a}}$ & 9 &, 000 \\
Razón de verosimilitud & 477,322 & 9 &, 000 \\
Asociación lineal por lineal & 243,668 & 1 &, 000 \\
N de casos válidos & 369326 & & \\
\hline
\end{tabular}

a. 0 casillas $(0,0 \%)$ han esperado un recuento menor que 5 . El recuento mínimo esperado es 1003,12 . 
Tabla 2. Relación estadística entre la variable nivel de estudios y tipo de parto

Medidas simétricas

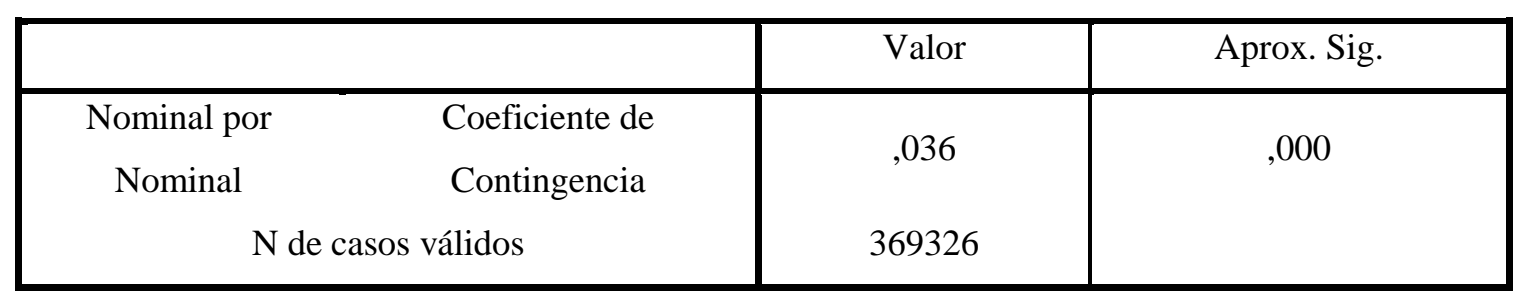

Fuente: Elaboración propia a partir de datos de nacimientos 2015. INE.

Otro de los indicadores sociodemográficos con los que se trabajó fue el del tipo de trabajo que desempeña la madre. La relación estadística medida con el coeficiente de contingencia en esta variable mostró que es significativa, mostrando que hay profesiones entre las que es más probable tener un parto por cesárea.

De hecho, a la vista de los porcentajes analizados, la profesión con el mayor porcentaje de parto por cesárea se encuentra entre las mujeres que trabajan en las fuerzas armadas, con un $38 \%$ del total de partos, o entre las que son pensionistas con un $34 \%{ }^{3}$ así como entre las directivas con el $31 \%$. Las profesiones entre las que el parto natural tiene el mayor porcentaje es el de estudiante con el $19 \%$ o la de tareas del hogar con el $24 \%$.

Las pruebas estadísticas muestran que es posible cierta dependencia entre la profesión y el tipo final de parto, tal como se puede comprobar en el test de contingencia y el Chi Cuadrado realizado entre las dos variables. En el análisis Chi Cuadrado, el contraste de la hipótesis muestra que la independencia no es significativa.

Tabla 3. Test de Chi Cuadrado de Pearson para la relación entre las variables entre tipo de trabajo y cesárea

Pruebas de chi-cuadrado

\begin{tabular}{|c|c|c|c|}
\hline & Valor & Gl & Sig. asintótica (2 caras) \\
\hline Chi-cuadrado de Pearson & $1072,560^{\mathrm{a}}$ & 12 &, 000 \\
Razón de verosimilitud & 1073,329 & 12 &, 000 \\
Asociación lineal por lineal & 522,838 & 1 &, 000 \\
N de casos válidos & 365692 & & \\
\hline
\end{tabular}

a. 0 casillas $(0,0 \%)$ han esperado un recuento menor que 5 . El recuento mínimo esperado es 381,80 .

\footnotetext{
${ }^{3}$ Mujeres que cobran algún tipo de ayuda por su situación de invalidez o discapacidad.
} 
Tabla 4. Relación estadística entre la variable tipo de trabajo de la madre y tipo de parto Medidas simétricas

\begin{tabular}{|c|c|c|c|}
\hline & & Valor & Aprox. Sig. \\
\hline $\begin{array}{c}\text { Nominal por } \\
\text { Nominal }\end{array}$ & $\begin{array}{l}\text { Coeficiente de } \\
\text { Contingencia }\end{array}$ & \multirow{2}{*}{$\begin{array}{c}, 054 \\
365692\end{array}$} & \multirow[t]{2}{*}{,000 } \\
\hline & ́́lidos & & \\
\hline
\end{tabular}

Fuente: Elaboración propia a partir de datos de nacimientos 2015. INE.

En lo que respecta al análisis de contingencia, se muestra que existe una relación de dependencia entre las dos variables, la del tipo de trabajo y el tipo de parto. En este sentido, ante la propuesta de introducir factores sociodemográficos para mejorar el perfil de prevención y atención de mujeres ante la situación de parto, encontramos que las mujeres trabajadoras, las cuales asumen situaciones de carga productiva y reproductiva (Durán, 1986), así como las situaciones de invalidez o discapacidad, son factores que pueden afectar a la hora de planificar un parto natural, ya que existe una cierta probabilidad de que termine siendo un parto por cesárea.

Por otra parte, el hecho de ser estudiante, factor correlacionado con la edad de la persona gestante, y el hecho de dedicarse la mujer únicamente al trabajo reproductivo producen que se pueda planificar con mayor antelación un parto natural, en tanto que el riesgo de cesárea es menor.

Si se toma como referencia la provincia en la que se produjo el parto, existe una elevada variabilidad entre las diferentes provincias. Así, en la provincia de Álava, únicamente el 13,4 \% de los partos terminan en cesárea y en Guipúzcoa el 17 \%, mientras que en el lado opuesto se sitúa Valencia, con el $33 \%$ de partos o Cáceres con el 32,2 \%. De este modo, la provincia en la que llega a término el parto condiciona el desenlace final del mismo.

Al analizar los test estadísticos, en lo que respecta a la variable «Provincia», es necesario exponer que no se da una relación unitaria e independiente en todo el estado, ya que existen diferencias cuando cruzamos los datos entre la provincia del nacimiento y el tipo de parto. Las pruebas muestran que no existe independencia entre las variables (en este caso mostraremos los datos en el mapa 1.). 
Mapa 1. Tasa de cesáreas por provincias. Nacimientos en 2015.

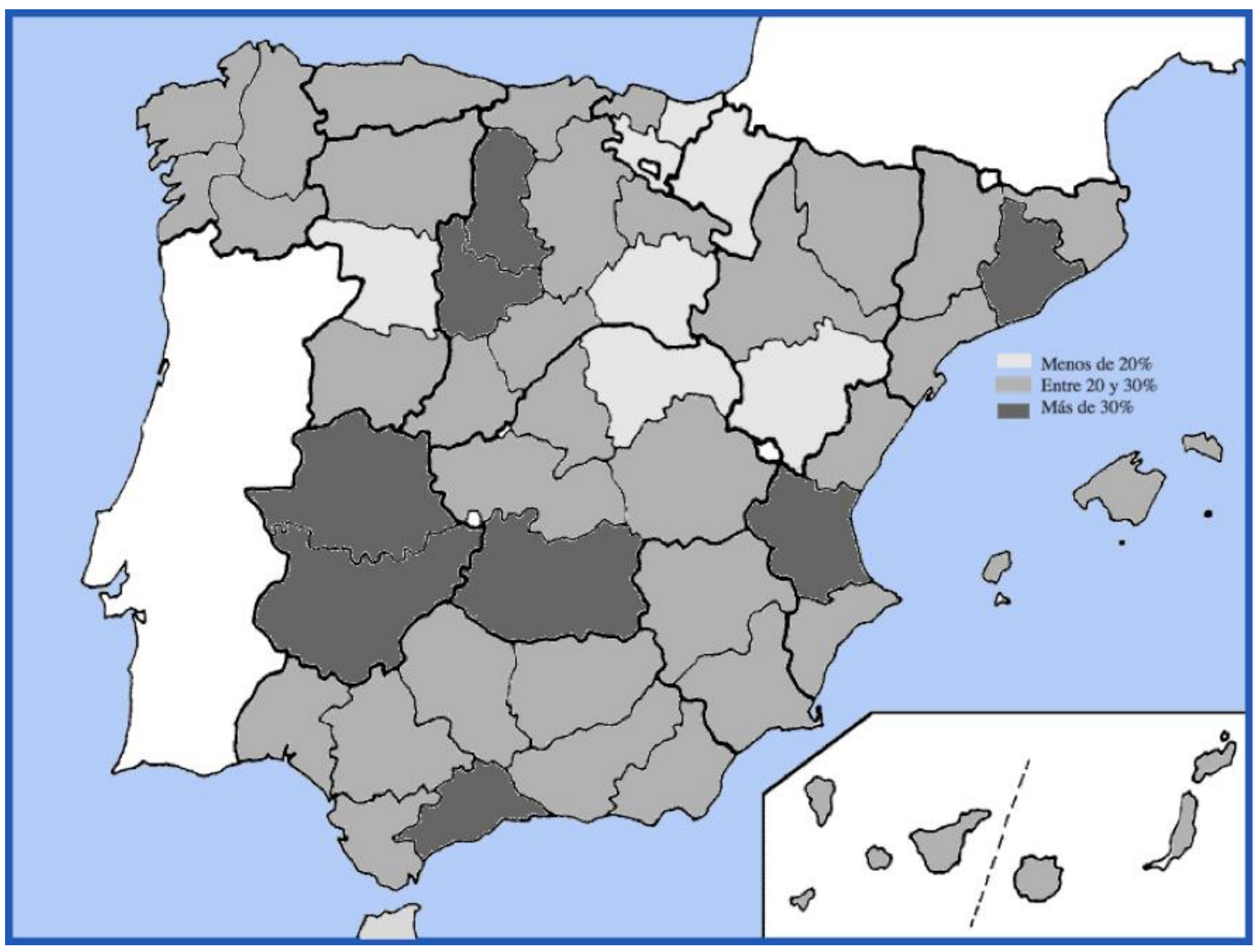

Fuente: Elaboración propia a partir de datos del INE de nacimientos de 2015.

En España en el 2015, al menos ocho provincias tenían una tasa de cesáreas por encima del $30 \%$, lo que significa que prácticamente uno de cada tres partos terminaba con una intervención quirúrgica.

En el extremo contrario, en otras siete provincias, la tasa se sitúa por debajo del 20\%, siendo, tal como hemos comentado, Álava la menor con el $13 \%$ del total y Valencia la que presentaba el porcentaje más alto con el $33 \%$.

Por todos los datos que se están mostrando, sería importante un estudio en profundidad de las causas que generan estos desajustes entre provincias. No parecen haber desajustes por Comunidades Autónomas, sino que más bien se trata de localizaciones provinciales.

\section{DISCUSIÓN}

En los debates médicos y académicos, se habla de la relación que existe entre diversos factores y la mayor o menor probabilidad de tener un parto por cesárea, como por ejemplo 
la preparación al parto, como señalan en sus investigaciones Patiño (2012), y Lepore, Sampor, Pardo y Pardo (2005).

De este modo, incluso ante los avances en bioética y medicina obstétrica familiar, el análisis de dichos factores son datos que no han sido incorporados todavía a las estadísticas que recoge el INE. Así, pues, sería de vital importancia para comprender mejor el proceso de parto, sobre todo por la baja natalidad del país que hace centrar la atención sobre estos temas. Es importante la elaboración de estudios que ayuden a incorporar poco a poco la multiplicidad de factores que envuelven a la mujer para completar con un hecho tan poliédrico como es la prevención de las cesáreas, configurando una mirada holística hacia un espacio que necesita de una continuada prevención y gestión de los procesos.

En el presente artículo, se trabaja con el nivel de estudios y profesión, que son los datos sociodemográficos que se recogen en las fichas de los nacimientos, y que, para autores como Pérez Díaz, son las variables sociodemográficas básicas (Pérez Díaz, Álvarez-Miranda y Chuliá Rodrigo, 1997), de pertinencia para la cobertura de las necesidades ante un proceso de gestación. De ahí que se haya considerado como argumento para una primera aproximación, dentro de la amplia multiplicidad de factores existentes, la relación entre variables sociodemográficas y tipo de parto. Tanto es así que a la vista de los datos se confirma que no existe una relación de independencia entre las variables.

En los estudios acerca de los motivos de las cesáreas, la perspectiva que han seguido las investigaciones se ha centrado principalmente en la preparación al parto o en factores vinculados con el tipo de hospital, etc. (Escuriet-Peiró et al., 2015). De este modo, partir de ciertos factores sociodemográficos nos aporta una nueva perspectiva para comprender si existe una relación estadística que se pueda tener en cuenta a la hora de trabajar con las mujeres en el preparatorio, atendiendo a las características demográficas.

Por otra parte, y siguiendo con el mismo argumento, junto a una primera aproximación estadística a los datos de nacimientos, sería importante realizar una investigación en mayor profundidad que partiese de las variables sobre las que se puede establecer una correlación estadística. En este sentido, cuando hablamos de partos por cesárea, volvemos a encontrar información más vinculada a los procesos médicos y a los resultados obtenidos, teniendo que destacar que los tipos de análisis realizados se han 
centrado en muchos casos en las causas médicas que pueden catalizar una cesárea. Así, pues, por la particularidad de los datos, y por su disponibilidad limitada, es por lo que la mayoría de los trabajos realizados han partido desde esta perspectiva de los propios hospitales, donde la información queda vinculada directamente con la proximidad al quehacer médico (Monterrosa-Castro y Árias-Martínez, 2007; Patiño, 2011).

De este modo, los análisis que se han venido realizando presentan los resultados exponiendo las causas que desencadenaron el desenlace de la cesárea, como una desproporción pélvico-cefálica, una cesárea anterior u otros factores (Patiño, 2011). Análogamente, otro de los factores analizados para comprobar la incidencia de la cesárea ha sido la obesidad de las embarazadas (Weiss et al., 2004), vinculados a las mismas razones médicas. También encontramos estudios que se han centrado en cómo los psicoprofilácticos pueden afectar al desarrollo del parto y a que este termine con una intervención quirúrgica (Márquez Calderón et al., 2011); o en la incidencia que podría tener la inducción al parto y el hecho de que este pudiera terminar en cesárea (OyuelaGarcía y Hernández-Herrera, 2010; Seyb et al., 1999).

Muy próxima a nuestra perspectiva se encuentra el texto de Martínez Frías et al., que muestra como factores como la edad del primer hijo pueden ser relevantes en el hecho de que el parto finalice con cesárea, así como la reproducción asistida puede llevar a producir un incremento exponencial de dichos tratamientos (Martínez-Frías et al., 2005). En el estudio, se ha comprobado que, a mayor edad, mayor es la probabilidad de cesárea.

De este modo, en el campo de la investigación de partos con cesáreas, vamos encontrando pequeños estudios que van desgranando poco a poco los factores sociodemográficos existentes. Un ejemplo de esto es el de Márquez-Calderón et al., en el cual se analizan los factores sociales del tipo de parto (Tessier et al., 2013). Sin embargo, cabe anotar que la perspectiva de estos autores se ha centrado en la descripción y no entra en la relación de dependencia entre variables desde la perspectiva estadística. El trabajo muestra la relación entre el parto con la edad y variables como la localización geográfica, y no parece haber relación entre las características demográficas de las zonas con la distribución de cesáreas por los territorios.

Una mención especial requieren las mujeres embarazadas de las fuerzas de seguridad del estado, sobre todo las militares, quienes poseen una elevada tasa de cesáreas. En este sentido, las militares tienen convenios con compañías aseguradoras privadas, por lo que 
la alta tasa de cesáreas entre las militares sería también una consecuencia de que en los hospitales privados la tasa de cesáreas es mayor. Otra explicación que requeriría una mayor profundidad a nivel cualitativo sería que las mujeres militares podrían planificarse los partos para mejorar su disponibilidad en el ejército y poder realizar maniobras o acciones en el exterior.

Este estudio no pretende realizar un análisis sistemático de las variables que pueden influir sobre las cesáreas en España, sino más bien llamar la atención al personal sanitario para que en los protocolos tengan en cuenta las características de la mujer, más allá del hecho evidente de que está embarazada y va a dar a luz. Así como la forma en la que se les explican las situaciones, puesto que si bien, a mayor edad, queda probada la mayor probabilidad de cesárea, como hemos mencionado, si los protocolos se adaptaran a las mujeres quizás el número de cesáreas se vería reducido. Y sería interesante en un estudio más pormenorizado la comprensión de si, en realidad, es la edad lo que determina o si bien depende de los tipos de trabajos que realizan.

Finalmente, cabe anotar que no solamente los factores sociodemográficos forman parte de los factores de prevención ante los posibles partos con cesáreas. Hay otros factores vinculados a marcos de interpretación cualitativos como los deseos, la planificación, las expectativas o el mismo proceso de preparación de la mujer y del hombre frente al parto. En este sentido, el siguiente paso que se está realizado desde esta investigación es ampliar el estudio a través de un estudio mediante metodología cualitativa con mujeres que han dado a los en los últimos cinco años, ${ }^{4}$ donde se está comprobando que las mujeres que asisten a las clases de preparación al parto tienen más información sobre las posibilidades del mismo, hecho que aporta una mejora en el proceso psicosocial de la paciente, así como en la toma de decisiones. De este modo, cabe entender la importancia de atender en toda su complejidad todos los procesos que atraviesan el parto, tanto desde la intervención médica, los efectos positivos o negativos a nivel de salud, pero también la prevención y los efectos que se derivan a nivel social.

\footnotetext{
${ }^{4}$ En el estudio que se menciona se están seleccionando mujeres que han dado a luz en los últimos cinco años, tanto en hospitales públicos como privados. En él se hace hincapié en el trato recibido por las mujeres, así como en la forma en la que se han sentido durante el parto y postparto. Destacan que una vez dan a luz existe el vacío. No hay acompañamiento y esto las deja en una situación de vulnerabilidad.
} 


\section{CONCLUSIONES}

En relación con el análisis cuantitativo realizado, esta aproximación a las variables sociodemográficas muestra la relevancia de trabajar con determinados perfiles sociales para mejorar las estadísticas en relación al número de cesáreas en España y de esta forma poder ajustarse un poco más a las recomendaciones de la OMS, ya que la salud de las mujeres gestantes y de las/los recién nacidos están en juego. No se trata únicamente de una cuestión de protocolos, sino de investigar con mayor profundidad por qué se está produciendo y cuáles son los factores que inciden en este fenómeno. Para ello, se hace imprescindible disponer de una investigación en la que se tome como referencia a grupos de mujeres en los que el seguimiento de su embarazo incluya sus variables sociales y sus pautas profesionales. Esto ayudaría a mostrar los posibles condicionantes en sus vidas antes y durante el embarazo y el futuro desenlace en el parto.

El estudio muestra que hay que prestar atención a los perfiles para implementar los trabajos de atención a las embarazas, pero para ello se necesitaría una investigación en mayor profundidad a fin de poder adecuar los protocolos de seguimiento ante las distintas realidades que se puedan dar e incidir en los motivos del por qué, a nivel estatal, en unas provincias existe tanta diferencia en el número de cesáreas realizadas a las mujeres.

Por otra parte, ante la diversidad de realidades dibujadas a raíz de los datos, para profundizar de forma más específica en la práctica de las cesáreas y la atención a los partos, es necesario que los y las profesionales que trabajan en torno a los procesos de la gestación sean capaces de escuchar y recoger las voces de las propias mujeres y que, en la medida de lo posible, ellas puedan volver a recuperar poder de decisión sobre sus propios cuerpos, empoderándose de nuevo en materia de salud sexual y reproductiva. Por esta razón, el siguiente paso lógico de este análisis sería acercarnos a la realidad de las mujeres, a sus voces, y profundizar en su experiencia, en los beneficios recibidos y en los malestares percibidos. 


\section{BIBLIOGRAFÍA}

Al AdIB MendiRI, Miriam et al. La violencia obstétrica: un fenómeno vinculado a la violación de los derechos elementales de la mujer. En: Medicina Legal de Costa Rica. 2017, pp. 104-11.

BELLón SÁNCHEZ, Silvia. La violencia obstétrica desde los aportes de la crítica feminista y la biopolítica. En: Dilemata. 2015, pp. 93-111.

BotToms, Sidney F.; Rosen, Mortimer G.; SokOL, Robert J. The increase in the cesarean birth rate. En: New England Journal of Medicine. 1980, pp. 559-63.

CERDÁN-JiMÉNEZ, María D. et al. Proceso de humanización de la atención a las cesáreas: instauración de un procedimiento para acompañamiento de las gestantes e inicio de la lactancia materna en quirófano. En: Musas. 2017, vol. 2, núm. 1, pp. 42-56.

Da Silva Carvalho, Isaiane; Santana De Brito, Rosineide. Formas de violencia obstétrica experimentada por madres que tuvieron un parto normal. En: Enfermería global: Revista Electrónica semestral de enfermería. 2017, pp. 71-79.

DURÁN, Ma Ángeles. La jornada interminable. Barcelona: Icaria Editorial, 1986.

ESCURIET-PEIRÓ, Ramón et al. Impact of maternity care policy in Catalonia: a retrospective cross-sectional study of service delivery in public and private hospitals. En: BMC Pregnancy and Childbirth. 2015, vol. 15, núm. 1, pp. 23.

FERNÁNDEZ GuILlÉN, Francisca. ¿Qué es la violencia obstétrica? Algunos aspectos sociales, éticos y jurídicos. En: Dilemata. 2015, pp. 113-28.

GARcíA, Eva Margarita. Hacia los partos empoderados: recuperando nuestros cuerpos secuestrados por la biomedicina. En: Dossiers Feministes. 2017, pp. 87-106.

GiJón, Natalia. Plan de Parto: nomenclatura, toma de decisiones e implicación de los profesionales sanitarios. En: Musas. 2016, vol. 1, núm. 2, pp. 35-51.

HADJIGEORGIOU, Eleni et al. Variation in caesarean section rates in Cyprus, Italy and Iceland: an analysis of the role of the media. En: Minerva Ginecologica. 2018, vol. 70, núm. 6, pp. 676-86.

HERAS, Blanca et al. La edad materna como factor de riesgo obstétrico. Resultados perinatales en gestantes de edad avanzada. En: Progresos de Obstetricia y Ginecología. 2011, pp. 575-80.

JOSEPH, K. S. et al. Changes in Maternal Characteristics and Obstetric Practice and Recent Increases in Primary Cesarean Delivery. En: Obstetrics \& Gynecology. 2003, pp. 791-800. 
MÁRQuez CALDERÓn, Soledad et al. Frecuencia de la cesárea en Andalucía: relación con factores sociales, clínicos y de los servicios sanitarios (2007-2009). En: Revista española de salud pública. 2011, pp. 205-15.

MARTÍNEZ-FríAS, María Luisa et al. Evolución secular y por autonomías de la frecuencia de tratamientos de fertilidad, partos múltiples y cesáreas en España. En: Medicina clínica. 2005, pp. 132-39.

Monterrosa-CAStro, Álvaro; Árias-MartíneZ, Merizalde. Partos vaginales y cesáreas en adolescentes: comportamiento entre 1993 y 2005 hospital de maternidad «Rafael Calvo», Cartagena (Colombia). En: Revista Colombiana de Obstetricia y Ginecología. 2007, pp. 107-14.

Nolasco-Ble, Anna K.; HernándeZ-Herrera, Ricardo J. \& RAMON-GonZÁleZ, René M. Hallazgos perinatales de embarazos en edad materna avanzada. En: Ginecología y Ostetricia de México. 2011, pp. 207-75.

ORgANIZACIÓN MUNDIAL DE LA SALUD. Recomendaciones de la OMS para los cuidados durante el parto, para una experiencia de parto positiva [en línea]. OMS, 2018 [en línea]. Disponible en:

<http://apps.who.int/iris/bitstream/handle/10665/272435/WHO-RHR-18.12spa.pdf?ua=1>.

Oyuela-García, Juana; Hernández-Herrera, Ricardo J. Psicoprofilaxis e índice de cesáreas. En: Revista Médica Instituto Mexicano Seguro Social. 2010, pp. 439-42.

PAtiÑo, Gladys. Factores determinantes de la cesárea en los hospitales de Tumbes. En: Ciencia y Desarrollo. 2012, pp. 31-7.

PÉREZ DíAZ, Victor; Álvarez-Miranda NAVARro, Berta; Chuliá Rodrigo, Elisa. La opinión pública ante el sistema de pensiones. Barcelona: Caja de Ahorros y Pensiones de Barcelona, 1997.

PROGRAMA DE NACIONES UnidAS PARA El DESARROLlo. ODS $n^{\circ}$ 5. Lograr la igualdad de género y el empoderamiento de las mujeres y las niñas [en línea]. 2015. Disponible en: 〈http://los17ods.org/los-17-objetivos-para-2030/igualdad-de-genero>.

SEYB, Stacy et al. Risk of cesarean delivery with elective induction of labor at term in nulliparous women. En: Obstetrics \& Gynecology. 1999, pp. 600-7.

TESSIER EsPAÑA, Esther et al. Cesárea humanizada. En: Progresos de obstetricia y ginecología. 2013, pp. 73-8. 
TIPIANI-RodRíGUEZ, Oswaldo. ¿Es la edad materna avanzada un factor de riesgo independiente para complicaciones materno-perinatales? En: Revista Peruana de Ginecología y Obstetricia. 2006, vol. 52, núm. 3, pp. 179-85.

VANINA, Gisela et al. Incidencia y principales indicaciones de la cesárea. En: Revista de Posgrado de la VIa Cátedra de Medicina. 2005, núm. 150, pp. 9-12.

VillarmeA, Stella; OlzA, Ibone; Recio, Adela. El Parto es Nuestro: el impacto de una asociación de usuarias en la reforma del sistema obstétrico de España. En: Dilemata. 2015, pp. 157-83.

WeISS, Joshua L. Obesity, obstetric complications and cesarean delivery rate-a population-based screening study. En: American Journal of Obstetrics and Gynecology. 2004, pp. 1091-7. 